\title{
Etude descriptive des caractéristiques morphométriques et génitales de la chèvre de Lubumbashi en République démocratique du Congo
}

\author{
I.A. Ngona ${ }^{1}$ J.M. Beduin ${ }^{2}$ \\ A.B.F. Khang'Maté ${ }^{1}$ C. Hanzen ${ }^{3 *}$
}

Mots-clés

Caprin - Chèvre - Mensuration corporelle - Genitalia - Abattoir République démocratique du Congo

\begin{abstract}
Résumé
L'étude a eu pour but de préciser les caractéristiques zootechniques et de reproduction des chèvres $(n=1311)$ et des boucs $(n=346)$ abattus à Lubumbashi. La taille au garrot, la longueur corporelle et le poids vif ont été respectivement de $53 \mathrm{~cm}, 77 \mathrm{~cm}$ et 20,5 kg chez les femelles, et de $53 \mathrm{~cm}, 71 \mathrm{~cm}$ et $19 \mathrm{~kg}$ chez les mâles. Le poids des testicules gauche et droit, le diamètre moyen des testicules et le périmètre scrotal ont été respectivement de $67,3 \mathrm{~g}$, $66,9 \mathrm{~g}, 12 \mathrm{~cm}$ et $21,1 \mathrm{~cm}$. La longueur moyenne du tractus génital de la vulve à l'extrémité de la corne a été de $30,3 \mathrm{~cm}$. Le poids moyen des ovaires, la longueur et la largeur des ovaires gauche et droit ont été respectivement de 1,1 $\mathrm{g}$, $1,8 \times 1,3 \mathrm{~cm}$ et 1,7 x 1,3 cm. Aucune structure kystique n'a été observée sur les ovaires. Quarante-quatre pourcent des femelles abattues se sont révélées gravides. A partir de la comparaison du nombre de corps jaunes et du nombre d'embryons ou de fœtus présents dans l'utérus, les mortalités embryonnaires ont été estimées à 19,2 p. 100.
\end{abstract}

\section{INTRODUCTION}

La population mondiale de caprins s'élevait en 2008 à 850 millions d'individus (16). L'extension croissante de l'élevage caprin dans les pays en développement ou émergents s'explique par le rôle essentiel qu'il exerce dans l'économie rurale en tant que ressource alimentaire et financière pour de nombreux petits éleveurs (3) du Katanga, notamment, dont 71 p. 100 de la population est sous-alimentée et 80 p. 100 vit en dessous du seuil de pauvreté (moins d'un dollar US par jour) (16). Qualifiée de « vache du pauvre », la chèvre s'adapte facilement à son environnement, ce qui en fait le principal ruminant dans les régions arbustives et les systèmes traditionnels extensifs des régions subtropicales (1).

Diverses études portant sur la reproduction des caprins ont montré l'importance majeure exercée par la croissance folliculaire, le

1. Université de Lubumbashi, faculté de Médecine vétérinaire, service de Reproduction, Obstétrique et Insémination artificielle, Lubumbashi, République démocratique du Congo.

2. Université de Liège, faculté de Médecine vétérinaire, Pharmacologie, Pharmacothérapie et Toxicologie, Liège, Belgique.

3. Université de Liège, faculté de Médecine vétérinaire, service de Theriogenologie des animaux de production, B 42 Sart Tilman, 4000 Liège, Belgique.

* Auteur pour la correspondance

E-mail : Christian.Hanzen@ulg.ac.be poids atteint au moment de la puberté ou encore la précocité et l'exactitude de constats précoces de gravidité, ou la sélection génétique sur l'optimisation des capacités de production et de reproduction des élevages caprins (3).

La présente étude a eu pour but de décrire les caractéristiques morphométriques et génitales de la race caprine Naine africaine majoritairement présente à Lubumbashi pour pouvoir à terme mettre en place un programme d'intensification de l'élevage familial.

\section{MATERIEL ET METHODES}

Les données concernent 1657 individus (1 311 femelles et 346 mâles) examinés ante et post mortem à l'abattoir de Lubumbashi durant la période comprise entre janvier 2006 et septembre 2007. Les animaux ont été abattus par leurs propriétaires pour des raisons plus souvent financières que zootechniques ou pathologiques.

Chaque individu a fait l'objet d'une caractérisation morphométrique : poids vif, état corporel, dentition, longueur occipito-coccygienne, hauteur au garrot, périmètre scrotal. Le poids de l'animal avant abattage et de la carcasse ont été relevés. L'état corporel a été évalué par palpation des vertèbres lombaires sur une échelle de notation à six points (de 0 état très maigre, à 5 état très gras). L'âge des animaux a été déterminé par examen de la dentition. 
Avant abattage des mâles, le testimètre a été enroulé autour de la partie médiane du scrotum, les testicules étant maintenus au fond des bourses. Après abattage, les testicules ont été prélevés sur la carcasse et pesés séparément. Les ovaires, oviductes et utérus (cornes, corps et col) ont fait l'objet d'un examen clinique pour identifier le statut de reproduction (gravide ou non) de chaque femelle, la présence de pathologies utérines et la nature des structures physiologiques ou pathologiques ovariennes. Les ovaires ont été pesés et leurs dimensions mesurées par échographie. La présence d'un corps jaune ou d'un follicule a été relevée par inspection visuelle et examen échographique des ovaires. Un follicule a été qualifié de kystique lorsque son diamètre était supérieur à $10 \mathrm{~mm}(9)$.

A l'examen échographique, le follicule est défini comme une structure anéchogène de diamètre supérieur compris entre 2 et $10 \mathrm{~mm}$ (14). Le corps jaune donne une image échographique plus isoéchogène. En l'absence de follicules et/ou de corps jaune, l'ovaire a été qualifié d'inactif. Le diamètre des cornes utérines a été déterminé par échographie et leur longueur au moyen d'une règle graduée.

Les statistiques descriptives ont été réalisées avec le logiciel R2.14.0 (4). Les données quantitatives ont été représentées par leurs valeurs moyennes et extrêmes, et les données qualitatives par leur nombre et pourcentage. La comparaison des moyennes a été faite grâce au test d'analyse de variance et celle des effectifs avec le test de Chi-carré de Pearson. La valeur $\mathrm{p}<0,05$ a été considérée comme significative.

\section{RESULTATS}

\section{Caractéristiques morphométriques}

La robe des 1657 individus était majoritairement de couleur noire et chamoisée. L'âge moyen des mâles et des femelles abattus a été respectivement de 17 et 38 mois. Une tendance significative ( $p<0,001)$ respectivement d'augmentation et de diminution de l'âge des femelles et des mâles abattus (tableau I) a été observée. Le pourcentage de femelles gravides abattues n'a pas été significativement influencé par l'âge $(\mathrm{p}>0,05)$.

Les valeurs moyennes et extrêmes (minimum et maximum) de la taille au garrot, de la longueur corporelle, du poids vif, du poids de la carcasse, du rendement à l'abattage et de l'état corporel des mâles et des femelles ont été comparables. Aucune différence significative en fonction des classes d'âge n'a été mise en évidence ( $p>0,05)$ (tableau II).

\section{Caractéristiques génitales}

Les poids et diamètres moyens des testicules gauche et droit sont apparus comparables, avec respectivement les valeurs de $67,3 \pm 17,04 \mathrm{~g}(25-136 \mathrm{~g})$ et $66,9 \pm 16,95 \mathrm{~g}(9-136 \mathrm{~g})$, et $11,96 \pm 1,11 \mathrm{~cm}(6,5-16 \mathrm{~cm})$ et $11,96 \pm 1,06 \mathrm{~cm}(7,4-15,8 \mathrm{~cm})$. Le périmètre scrotal moyen des deux testicules a été de $21 \pm 2,39 \mathrm{~cm}(6-27 \mathrm{~cm})$. Les classes d'âge n'ont pas présenté de différence significative. Il en a été de même entre testicules gauche et droit $(\mathrm{p}>0,05)$.

Chez la chèvre non gravide, la longueur moyenne du tractus génital mesuré de la vulve à l'extrémité des cornes a été de $30,3 \mathrm{~cm}$. Le ligament intercornual a rendu les cornes jointives sur une longueur de 5,3 $\pm 1,45 \mathrm{~cm}(1,6-9,7 \mathrm{~cm})$. La longueur moyenne de la commissure vulvaire a été de $2,8 \pm 0,44 \mathrm{~cm}(2,3-3,1 \mathrm{~cm})$. Les longueurs moyennes du vestibule vulvaire, vagin, col, corps et cornes utérines ont été respectivement de $3,9 \pm 1,2 \mathrm{~cm}(2,3-6,0 \mathrm{~cm})$, $6,2 \pm 1,2 \mathrm{~cm}(4,1-9,4 \mathrm{~cm}), 3,3 \pm 0,9 \mathrm{~cm}(1,0-6,2 \mathrm{~cm}), 1,9 \pm 0,6 \mathrm{~cm}$ $(0,6-3,6 \mathrm{~cm})$ et $15 \pm 3,2 \mathrm{~cm}(6,1-23,4 \mathrm{~cm})$.

L'examen des utérus a permis d'identifier 43,6 p. 100 de femelles gravides avec une prolificité moyenne de 1,5. Lors de gravidité simple, la fréquence de la gravidité s'est avérée comparable dans les cornes gauche (47 p. 100) et droite (53 p. 100). Lors de

\section{Tableau I}

Distribution de l'effectif par classe d'âge, sexe et état de gravidité

\begin{tabular}{lrrrr}
$\begin{array}{l}\text { Age } \\
\text { (mois) }\end{array}$ & $\begin{array}{c}\text { Total mâles } \\
\text { et femelles } \\
\text { N }\end{array}$ & Mâles & Femelles & \multicolumn{1}{c}{$\begin{array}{c}\text { Femelles } \\
\text { gravides * } \\
\text { N }(\%)\end{array}$} \\
\hline $7-14$ & 281 & $177(63,0)$ & $104(37,0)$ & $35(33,6)$ \\
$15-20$ & 310 & $90(29,0)$ & $220(71,0)$ & $93(42,2)$ \\
$21-25$ & 203 & $27(13,3)$ & $176(86,7)$ & $69(39,2)$ \\
$26-35$ & 301 & $20(6,6)$ & $281(93,4)$ & $138(49,1)$ \\
$36-83$ & 306 & $16(5,2)$ & $290(94,8)$ & $130(44,8)$ \\
$84-107$ & 59 & $2(3,4)$ & $57(96,6)$ & $30(52,6)$ \\
$\geq 108$ & 197 & $14(7,1)$ & $183(92,9)$ & $77(42,0)$ \\
\hline Total & 1657 & 346 & 1311 & 572 \\
\hline
\end{tabular}

* Le pourcentage de femelles gravides a été calculé par rapport au nombre total de femelles

\section{Tableau II}

Caractéristiques morphométriques et zootechniques des boucs et des chèvres






\section{Tableau III}

Comparaison des poids et dimensions de l'ovaire des femelles gravides et non gravides

\begin{tabular}{|c|c|c|c|c|c|c|}
\hline & \multicolumn{2}{|c|}{ Gravides } & \multicolumn{2}{|c|}{ Non gravides } & \multicolumn{2}{|c|}{ Total } \\
\hline & $\mathbf{N}$ & Moyenne & $\mathbf{N}$ & Moyenne & $\mathbf{N}$ & Moyenne \\
\hline Poids ovaire G (g) & 117 & $1,3 \pm 0,58^{a}$ & 228 & $1,0 \pm 0,46^{b}$ & 345 & $1,1 \pm 0,52$ \\
\hline Poids ovaire D (g) & 111 & $1,4 \pm 0,57^{a}$ & 232 & $1,0 \pm 0,44 b$ & 343 & $1,1 \pm 0,52$ \\
\hline Longueur ovaire G (cm) & 500 & $1,9 \pm 0,42^{a}$ & 617 & $1,7 \pm 0,43 b$ & 1117 & $1,8 \pm 0,43$ \\
\hline Largeur ovaire G (cm) & 500 & $1,3 \pm 0,26^{a}$ & 617 & $1,3 \pm 0,27$ & 1117 & $1,3 \pm 0,27$ \\
\hline Longueur ovaire D (cm) & 473 & $1,8 \pm 0,41^{a}$ & 613 & $1,6 \pm 0,41 b$ & 1086 & $1,7 \pm 0,41$ \\
\hline Largeur ovaire D (cm) & 473 & $1,4 \pm 0,28^{a}$ & 613 & $1,3 \pm 0,27 b$ & 1086 & $1,3 \pm 0,27$ \\
\hline
\end{tabular}

G : gauche ; D : droit

${ }^{a}$ et ${ }^{b}$ Valeurs significativement différentes $(\mathrm{p}<0,001)$

\section{Tableau IV}

Distribution comparée des structures ovariennes chez les femelles gravides et non gravides

\begin{tabular}{|c|c|c|c|c|c|c|c|c|c|c|}
\hline \multirow[t]{2}{*}{ Ovaires des femelles } & \multicolumn{2}{|c|}{ Total } & \multicolumn{2}{|c|}{10} & \multicolumn{2}{|c|}{$\mathbf{F}$} & \multicolumn{2}{|c|}{ CJ } & \multicolumn{2}{|c|}{ Total F et CJ } \\
\hline & $\mathbf{N}$ & $\%$ & $\mathbf{N}$ & $\%$ & $\mathbf{N}$ & $\%$ & $\mathbf{N}$ & $\%$ & $\mathbf{N}$ & $\%$ \\
\hline Gravides & 1386 & 42,8 & 152 & 31,1 & 393 & 27,3 & 841 & 64,3 & 1234 & 44,9 \\
\hline Non gravides & 1851 & 57,2 & 338 & 68,9 & 1047 & 72,7 & 466 & 35,7 & 1513 & 55,1 \\
\hline Total & 3237 & 100 & 490 & 100 & 1440 & 100 & 1307 & 100 & 2747 & 100 \\
\hline
\end{tabular}

IO : inactivité ovarienne ; absence de corps jaune ou de follicules de diamètre supérieur à $2 \mathrm{~mm}$

$\mathrm{F}$ : follicules de diamètre compris entre 2 et $10 \mathrm{~mm}$

CJ : corps jaune

gravidité multiple, les embryons et/ou fœus ont été également distribués dans les deux cornes. Les différences observées n'ont pas été significatives $(p>0,05)$. La détermination du sexe fœtal a montré que la sex-ratio était de 0,53. L'âge moyen des femelles gravides n'a pas été significativement différent de celui des femelles non gravides (38,8 vs 37,6 mois).

Les poids, longueurs et largeurs moyens des ovaires des femelles sont apparus significativement plus élevés chez les animaux gravides que non gravides ( $\mathrm{p}<0,001)$ (tableau III).

Les examens visuels et échographiques de 3237 ovaires (tableau IV) a permis de constater la présence d'une structure folliculaire et/ ou lutéale dans 84,9 p. 100 des cas. Ces structures ont davantage été de type folliculaire $(\mathrm{n}=1440 ; 52,4$ p. 100) que lutéal $(\mathrm{n}=$ 1307 ; 47,6 p. 100). Le nombre moyen de structures folliculaires et/ou lutéales par ovaire a été de 1,3. Il s'est révélé légèrement supérieur chez les chèvres non gravides $(1,2)$ que gravides $(1,1)$. Une structure folliculaire a davantage été observée sur les ovaires des femelles non gravides $(\mathrm{n}=1047 ; 72,7$ p. 100) que gravides ( $\mathrm{n}=393 ; 27,3$ p. 100). A l'inverse, un corps jaune a davantage été observé sur les ovaires des femelles gravides $(n=841 ; 64,3$ p. 100) que non gravides $(\mathrm{n}=466 ; 35,7)$. Dans 79,4 p. 100 des cas, un seul corps jaune a été constaté sur les ovaires. La présence simultanée de deux et de trois corps jaunes a été observée dans respectivement 17,2 et 3,4 p. 100 des cas. La présence de 1, 2 et 3 corps jaunes a été observée dans respectivement 77,6, 18,5 et 3,9 p. 100 des cas. Une comparaison du nombre de corps jaunes et d'embryons ou de

\section{Tableau V}

Comparaison entre ovaires des diamètres moyens des follicules et corps jaune (en $\mathrm{mm}$ ) chez la chèvre gravide et non gravide

\begin{tabular}{lcccc} 
& \multicolumn{2}{c}{ Follicule } & \multicolumn{2}{c}{ Corps jaune } \\
\cline { 2 - 3 } & Gravide & Non gravide & Gravide & Non gravide \\
N & 393 & 958 & 835 & 380 \\
Moyenne & 4,3 & 4,1 & $9,7^{a}$ & $7,9 \mathrm{~b}$ \\
Ecart-type & 1,1 & 1,4 & 2,2 & 2,2 \\
Minimum & 1,5 & 1,1 & 3 & 2,1 \\
Maximum & 10 & 10 & 20 & 17,5
\end{tabular}

a et ${ }^{b}$ Valeurs significativement différentes $(\mathrm{P}<0,0001)$

fœetus a été réalisée sur 459 chèvres gravides. Dans 19,2 p. 100 des cas, le nombre de corps jaunes a été supérieur au nombre d'embryons ou de fœtus présents dans les cornes $(\mathrm{p}<0,001)$.

Aucune différence significative du diamètre moyen du follicule (4,2 mm) n'a été observée entre les chèvres gravides et non gravides $(p>0,05)$. Le diamètre moyen du corps jaune des chèvres non gravides a été significativement plus petit que celui des chèvres gravides $(\mathrm{p}<0,0001)$ (tableau $\mathrm{V})$. 


\section{DISCUSSION}

La caractérisation phénotypique d'une race (5) constitue la première étape indispensable à son amélioration génétique. La détermination de la taille au garrot et du poids des animaux de l'échantillon de l'étude a incité les auteurs à les classer dans la catégorie des caprins de petit gabarit. En effet, la taille moyenne au garrot observée $(53 \mathrm{~cm} ; 39-66 \mathrm{~cm})$ a été légèrement supérieure à celle de la chèvre West African Dwarf (WAD) observée au Bénin $(48,2 \mathrm{~cm})$ (5) et au Burkina Faso $(48,8 \mathrm{~cm})(15)$, mais elle a été nettement inférieure à celle de la chèvre de Mashona, également appelée East African, au Zimbabwe $(56,7 \mathrm{~cm})(11)$, et à celle de la chèvre rousse de Maradi $(64,2-68 \mathrm{~cm})(8)$. Le poids moyen des animaux de la présente étude $(20,5 \mathrm{~kg} ; 7,9-41,1 \mathrm{~kg})$ a été inférieur à ceux observés pour la WAD en Gambie $(24,5 \mathrm{~kg} ; 20-30 \mathrm{~kg})(2)$ et pour la East African (27 kg ; 26,3-27,7 kg) (11). Compte tenu de la méthode utilisée, les observations de la présente étude ne peuvent être étendues à l'ensemble de la population. Elles peuvent néanmoins être considérées comme représentatives en raison, d'une part, du nombre d'animaux concernés et, d'autre part, du fait que cet échantillon comportait un pourcentage élevé d'animaux gravides, situation peu compatible avec une décision de réforme pour des raisons pathologiques ou nutritionnelles. Le périmètre scrotal moyen du bouc de Lubumbashi a été inférieur à celui rapporté pour la race Rayini en Iran (17).

Les données relatives aux caractéristiques pondérales et de taille des ovaires des races caprines évoluant en milieu tropical et subtropical sont encore peu nombreuses. Le cas échéant, elles n'ont été déterminées que sur quelques dizaines d'individus. Le poids moyen des ovaires de la chèvre de Lubumbashi $(1,1 \mathrm{~g})$ a été comparable à celui relevé pour la chèvre Black Bengal, soit 0,66 à $0,9 \mathrm{~g}$ pour l'ovaire droit et 0,64 à $0,85 \mathrm{~g}$ pour l'ovaire gauche (7), mais il a été inférieur à celui observé chez la chèvre locale iranienne par Mohammad Pour (1,98 à 2,16 g) (10), et par Rondina et coll. $(0,94$ à 1,48 g) (13). Ces derniers ont par ailleurs constaté un impact négatif de la sous-nutrition sur le poids moyen des ovaires, celui-ci étant respectivement compris entre 1,45 et 1,48 g, et entre 0,94 et $1,03 \mathrm{~g}$ chez les femelles correctement ou non alimentées (13).

La comparaison du nombre de corps jaunes à celui des embryons et/ou fœtus identifiés dans l'utérus a permis d'estimer à 19,2 p. 100 la mortalité embryonnaire. Ce pourcentage est comparable à celui observé chez la chèvre du Sahel (19,7 p. 100) (6).

Les mesures échographiques de la longueur des embryons et/ ou des fotus a permis d'observer que la majorité $(55$ p. 100) des femelles gravides a été abattue durant la deuxième moitié de la gravidité. Les résultats ont montré que 44 p. 100 des chèvres abattues étaient gravides. L'importance de ces pertes confirme la nécessité et l'intérêt d'une identification échographique des femelles avant leur abattage. Le manque de collecte de données pour les élevages concernés est une carence pouvant être surmontée par l'organisation de l'élevage local. Ceci éviterait de réformer des femelles gravides et contribuerait à une bonne gestion de la reproduction caprine et à une bonne rentabilité des individus.

\section{$\square$ CONCLUSION}

Cette étude a permis de préciser les caractéristiques de développement et génitales des caprins de Lubumbashi. Elles sont assimilables à la race Naine de cette espèce. Une activité ovarienne a été observée chez la majorité des chèvres étudiées. Cela laisse entrevoir la possibilité d'une mise en place de traitements de synchronisation pour améliorer la productivité de ces femelles. La fréquence élevée de chèvres gravides autorise à recommander un dépistage plus systématique par échographie avant l'abattage, qui permettrait d'améliorer les revenus des éleveurs.

\section{Remerciements}

Nous remercions la Commission universitaire pour le développement dont le financement a permis différents stages à l'université de Liège pour le traitement des données.

\section{BIBLIOGRAPHIE}

1. ALEXANDRE G., MANDONNET N., 2005. Goat meat production in harsh environments. Small Rumin. Res., 60: 53-66.

2. BOSSO N.A., CISSE M.F., VAN DER WAAIJ E.H., VAN ARENDONK J.A.M., 2007. Genetic and phenotypic parameters of body weight in West African Dwarf goat and Djallonke sheep. Small Rumin. Res., 67: 271-278.

3. BOYAZOGLU J., HATZIMINAOGLOU I., MORAND-FEHR P., 2005. The role of the goat in society: past, present and perspectives for the future. Small Rumin. Res., 60: 13-23.

4. DEVELOPMENT CORE TEAM R., 2010. A language and environment for statistical computing. Vienna, Austria, R Foundation for Statistical Computing. http://www.R-project.org

5. DOSSA L.H., WOLLUY C., GAULY M., 2007. Spatial variation in goat population from Benin as revealed by multivariate analysis of morphological traits. Small Rumin. Res., 73: 150-159.

6. GNANDA B.I., ZOUNDI S.J., NIANOGO J.A., MEYER C., ZONO O., 2005. Test d'un complément minéral et azoté sur les paramètres de reproduction de la chèvre du Sahel burkinabé. Rev. Elev. Méd. vét. Pays trop., 58 : 257-265.

7. ISLAM M.R., KHANDOKER M.M.A.M.Y., AFROZ S., RAHMAN M.G.M., KHAN R.I., 2007. Qualitative and quantitative analysis of goat ovaries, follicles and oocytes in view of in vitro production of embryos. J. Zhejiang Univ. Sci B, 8: 464-469.

8. MARICHATOU H., MAMANE L., BANOIN M., BARIL G., 2002. Performances zootechniques des caprins au Niger : étude comparative de la chèvre rousse de Maradi et de la chèvre à robe noire dans la zone de Maradi. Rev. Elev. Méd. vét. Pays trop., 55 : 79-84.

9. MEDAN M., WATANABE G., SASAKI K., TAYA K., 2004. Transrectal ultrasonic diagnosis of ovarian follicular cysts in goats and treatment with GnRH. Domest. Anim. Endocrinol., 27: 115-124.

10. MOHAMMAD POUR A.A., 2007. Comparative histomorphological study of ovary and ovarian follicles in Iranian Lori-Bakhtiari sheep and native goat. Pak. J. Biol. Sci., 10: 673-675.

11. NDLOVU L.R., SIMELA L., 1996. Effect of season of birth and sex of kid on the production of live weaned single born kids in smallholder East African goat flocks in North East Zimbabwe. Small Rumin. Res., 22: 1-6.

12. QI Y., LUO J., HAN X.F., ZHU Y.Z., CHEN C., LIU J.X., SHENG H.J., 2009. Genetic diversity and relationships of ten chinese goat breeds in Middle and Western China. Small Rumin. Res., 82: 88-89.

13. RONDINA D., FREITAS V.J.F., SPINACI M., GALEATI G., 2005. Effect of nutrition on plasma progesterone levels, metabolic parameters and small follicles development in unstimulated goats reared under constant photoperiod regimen. Reprod. Domest. Anim., 40: 548-552.

14. SIMOES J., POTES J., AZEVEDO J., ALMEIDA J.C., FONTES P., BARIL G., MASCARENHAS R., 2005. Morphometry of ovarian structures by transrectal ultrasonography in Serrana goats. Anim. Reprod. Sci., 85: 263273.

15. TRAORE A., TAMBOURA H.H., KABORE A., ROYO L.J., FERNANDEZ I., ALVAREZ I., SANGARE M., BOUCHEL D., POIVEY J.P., FRANCOIS D., SAWADOGO L., GOYACHE F., 2008. Multivariate analyses on morphological traits of goats in Burkina Faso. Arch. Tierz., Dummerstorf, 51: 588-600

16. VANDENPUT S., 2006. Contribution à la satisfaction des besoins économiques et alimentaires de la population lushoise par le développement d'un élevage caprin intégré aux activités agricoles périurbaines. Projets interuniversitaires ciblés en matière de coopération au développement. Liège, Belgique, Faculté universitaire des Sciences agronomiques de Gembloux, p. 43.

17. ZAMIRI M.J., HEIDARI A.H., 2006. Reproductive characteristics of Rayini male goats of Kerman province in Iran. Anim. Reprod. Sci., 96: 176-185.

Accepté le 13.02.2013 


\section{Summary}

Ngona I.A., Beduin J.M., Khang'Maté A.B.F., Hanzen C. Descriptive study of the morphometric and genital characteristics of goats in Lubumbashi in the Democratic Republic of Congo

The study aimed to determine zootechnical and reproduction characteristics of female $(n=1311)$ and male $(n=346)$ goats slaughtered in Lubumbashi. Height at withers, body length and live weight were $53 \mathrm{~cm}, 77 \mathrm{~cm}$ and $20.5 \mathrm{~kg}$ in females, and $53 \mathrm{~cm}, 71 \mathrm{~cm}$ and $19 \mathrm{~kg}$ in males, respectively. The left and right testicular weights, diameter of testes and scrotal perimeter were $67.3 \mathrm{~g}$, $66.9 \mathrm{~g}, 12 \mathrm{~cm}$ and $21.1 \mathrm{~cm}$, respectively. The mean length of the genital tract from the vulva to the end of the horn was $30.3 \mathrm{~cm}$. The mean weight of ovaries, length and width of left and right ovaries were $1.1 \mathrm{~g}$, $1.8 \times 1.3 \mathrm{~cm}$ and $1.7 \times 1.3 \mathrm{~cm}$, respectively. No cystic structure was observed on the ovaries. Forty-four percent of the slaughtered females were found pregnant. From the comparison of the number of corpora lutea and of embryos or fetuses in the womb, embryonic mortality was estimated at $19.2 \%$.

Keywords: Goat - Body measurement - Genitalia - Abattoir Democratic Republic of Congo.

\section{Resumen}

Ngona I.A., Beduin J.M., Khang'Maté A.B.F., Hanzen C. Estudio descriptivo de las características morfométricas y genitales de la cabra de Lubumbashi en la República democrática de Congo

El estudio tiene como objetivo el de precisar las características zootécnicas y de reproducción de las cabras ( $\mathrm{n}=1311$ ) y machos cabríos $(\mathrm{n}=346)$ sacrificados en Lubumbashi. El tamaño al garrote, la longitud corporal y el peso vivo fueron respectivamente de $53 \mathrm{~cm}, 77 \mathrm{~cm}$ y $20,5 \mathrm{~kg}$ en las hembras, y de $53 \mathrm{~cm}, 71 \mathrm{~cm}$ y $19 \mathrm{~kg}$ en los machos. El peso de los testículos izquierdo y derecho, el diámetro promedio de los testículos y el perímetro escrotal fueron respectivamente de $67,3 \mathrm{~g}, 66,9 \mathrm{~g}, 12 \mathrm{~cm}$ y $21,1 \mathrm{~cm}$. La longitud promedio del tracto genital medido de la vulva a la extremidad del cuerno fue de $30,0 \mathrm{~cm}$. El peso promedio de los ovarios, la longitud y el ancho de los ovarios izquierdo y derecho fueron respectivamente de 1,1 g, 1,8 x 1,3 cm y 1,7 x 1,3 cm. No se observó ninguna estructura quística en los ovarios. Cuarenta y cuatro por ciento de las hembras sacrificadas estaban preñadas. A partir de la comparación entre el número de cuerpos lúteos y el número de embriones o de fetos presentes en los úteros, la mortalidad embrionaria fue estimada a 19,2\%.

Palabras clave: Caprino - Cabra - Medición del cuerpo Genitalia - Matadero - República Democrática del Congo. 
